Pacific Journal of Mathematic 


\section{ON WEAK DIMENSION OF ALGEBRAS}

\section{ORLANDO E. Villamayor}

1. Introduction. In this note we try to characterize algebras whose weak dimension is zero, i.e., algebras $A$ which are flat $A^{e}$-modules.

In this direction, Theorems 1 and 2 give the corresponding results, for weak dimension, to known theorems for (strong) dimension. However, it seems to be more interesting to find relations between these two dimensions.

Theorem 3 gives such a relation for commutative algebras over a field. For the non-commutative case, only a weaker necessary condition is found in Theorem 5. However, in the case of algebras satisfying the descending chain condition for left ideals a complete picture of the 0 -weak dimensional ones is given in Theorem 6 .

Section 6 applies these results to group algebras. In [2] Auslander partially succeded in characterizing (von Neumann) regular group algebras. However, concerning the group, he only proved the necessity of the group being torsion and the sufficiency of the local finiteness. The difference seemed to be related to the Burnside problem. Theorem 8, then, fills the gap and the problem is now completely solved.

In the last section we study some relaitons between weak dimensionality and semisimplicity (in the sense of Jacobson) in tensor products of algebras.

After this paper was written we received a copy of a paper by Prof. Harada on the same subject [4]. However, there is no overlapping of the main results.

We would like to express our thanks and indebtedness to Professor Rosenberg for this helpful advice and criticism.

2. Notations and terminology. Throughout this note we use the homological notation and terminology of [2].

Since we are dealing with algebras over a (fixed) ground ring $K$, all tensor products are suppose to be taken over the ground ring $K$, unless otherwise specifically expressed, so, we shall use $\otimes$ for $\otimes_{K}$. Similarly, homological dimension of algebras are indicated by $\operatorname{dim} A$ or w.dim. $A$ if they are considered over $K$, or $R$-dim $A$ (resp. $R$-w.dim $A$ ) if they are considered over another ring $R$.

For a ring, simple and semisimple mean simple and semisimple with minimum condition for one-sided ideals. Regular will always mean regular in the sense of von Neumann.

Received October 10, 1958, and in revised form February 9, 1959. This work was partially supported by a J. S. Guggenheim fellowship and by the Office of Naval Research. 
Semisimplicty in the sense of Jacobson is called $J$-semisimplicity.

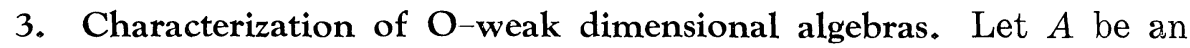
algebra over a commutative ring $K$. The dimension (resp. weak dimension) of $A$ as an algebra is, following the classical definitions, the dimension (resp. w.dim) of $A$ as an $A^{e}$-module, where $A^{e}=A \otimes A^{*}\left(A^{*}\right.$ the algebra anti-isomorphic to $A$ ). Since $A$ is a cyclic $A^{\rho}$-module, we shall start with some considerations on cyclic flat modules (i.e. cyclic modules $M$ with w.dim. $M=O$ ).

Lemma 1. Let $R$ be any ring and $A$ a cyclic left $R$-module. Then the following conditions are equivalent:

(a) $A$ is $R$-flat.

(b) $\operatorname{Tor}_{1}^{R}(R / I, A)=O$ for every principal right ideal $I$ in $R$.

(c) If $A=R / J$, a the image of 1 in $A$ and $x \in J$, there exists $y \in R$ such that $x y=O, y a=a$.

Proof. $\quad(a) \Rightarrow(b)$ is obvious.

(b) $\Rightarrow(c)$. Let $x \in J$ and let $I$ be the right ideal generated by $x$. According to ([3], VI, Ex. 19, p. 126) condition (b) implies $I \cap J=I J$. Since $x \in I \cap J$, then $x \in I J$, that is, there is a $z \in J$ such that $x=x z$, hence $z a=O$, and $y=1-z$ verifies $x y=O$ and $y a=a$. (c) $\Rightarrow$ (a). Let $B=R / I$, for any right ideal $I$. If $x \in I \cap J$, condition (c) assures the existence of an element $z \in J$ such that $x z=x$, so that $x \in I J$, hence $I \cap J=I J$. That is, $\operatorname{Tor}_{1}^{R}(B, A)=O$ for every cyclic module $B$ ([3], VI, Ex. 19), so $A$ is flat ([5]).

As a consequence, we obtain

Lemma 2. Let $A=R / J$ be a cyclic flat left-R-module. If I is a finitely generated left ideal contained in $J$, there is a principal left ideal $I^{\prime}$ such that $I \subseteq I^{\prime} \subseteq J$.

Proof. We proceed by induction on the number of generators of $I$. If $I$ has one generator, $I=I^{\prime}$. Suppose the lemma is true if $I$ has $n-1$ generators, and suppose $x_{1}, \cdots, x_{n}$ generate $I$. Let us call $a$ the image of 1 in $A$. If $x_{1}, x_{2} \in I \subseteq J$, then $x_{1} a=x_{2} a=O$ and there is an element $y \in R$ such that $x_{1} y=O$ and $y a=a$, hence $x_{2} y a=O$, and there is a $z \in R$ such that $x_{2} y z=O$ and $z a=a$, so $y z a=a$. If we call $r=$ $1-y z$ then $x_{1} r=x_{1}, x_{2} r=x_{2}$ and $r a=O$. This last condition implies $r \in J$ and $I \subseteq I_{1} \subseteq J$ where $I_{1}$ is the ideal generated by $r, x_{3}, \cdots, x_{n}$.

From these lemmas, the following well known result may be immediately proved:

Corollary 1. If a cyclic left module $A=R / I$ is $R$-flat and $I$ is 
finitely generated, then $A$ is $R$-projective.

In fact, Lemma 2 implies $I$ is generated by a single element, say $x$, and Lemma 1 assures the existence of $y \in I$ with $x y=x$, hence $R \rightarrow R y$ is a projection of $R$ onto $I$ and $I$ is a direct summand in $R$, so $A$ is projective.

Now, we shall apply these results to characterize $O$-weak dimensional algebras and the following theorem corresponds to that one given in [3] (IX, Proc. 7.7, p, 179) for dim. $A=O$.

THEOREM 1. In order that w.dim $A=0$ it is necessary and sufficient that, for every finite set $\left\{a_{1}, \cdots, a_{n}\right\}$ in $A$, there exists an element $e$ in the two-sided $A$-module $A \otimes A$ such that $a_{i} e=e a_{i}(1 \leq i \leq n)$ and that, under the mapping $x \otimes y \rightarrow x y$ the image of $e$ in $A$ is 1 .

Proof. Let $a_{1}, \cdots, a_{n} \in A$. Suppose w.dim $A=O$, i.e., $A$ is $A^{e}$-flat. The elements $1 \otimes a_{i}^{*}-a_{i} \otimes 1^{*}$ belong to $J=\operatorname{Ker}\left(A^{e} \rightarrow A\right)$, then they are contained in a principal left-ideal $I \subseteq J$. If $z$ is the generator of $I, z .1=O(1 \in A)$, then there is an element $e$ such that $e .1=1$ and $z e=O$, hence $\left(1 \otimes a_{i}^{*}-a_{i} \otimes 1^{*}\right) e=O$ and the necessity of the conditions proved.

To prove the sufficiency, let us consider an element $z \in J$. Thus $z=\sum y_{i}\left(1 \otimes a_{i}^{*}-a_{i} \otimes 1\right)\left(y_{i} \in A^{e}, a_{i} \in A\right)$, so, there is an $e \in A^{e}$ such that $\left(1 \otimes a_{i}^{*}-a_{i} \otimes 1\right) e=O, e .1=1$, hence $z e=O$ and Lemma 1 implies $A$ is $A^{e}$-flat.

As a consequence of Theorem 1 and [3], (IX, prop. 7.7) we obtain

Corollary 2. If $A$ is a finitely generated $K$-algebra, then w.dim $A=$ 0 if and only if $\operatorname{dim} . A=0$.

Of course, this result may also be obtained from Corollary 1 and the fact that $\operatorname{Ker}\left(A^{e} \rightarrow A\right)$ is a left ideal generated by the set $\left\{a_{i} \otimes 1^{*}-1 \otimes a_{i}^{*}\right\}$, where the $a_{i}$ 's generate $A$ as an algebra.

Now, following the same lines given by Rosenberg and Zelinsky ([9], Th. 1, p. 88) we prove

Theorem 2. Let $A$ be a K-algebra which is free as a K-module. If $\mathrm{w} \cdot \operatorname{dim} A=O$, then $A$ is locally finite over $K{ }^{1}$

Proof. Let $\left\{x_{i}\right\}$ be a $K$-basis of $A$ and $\left\{b_{1}, \cdots, b_{n}\right\}$ be a finite subset in $A$. If $B$ is the subalgebra generated by the set $\left\{b_{1}, \cdots, b_{n}\right\}$, then, for every $z \in B, 1 \otimes z^{*}-z \otimes 1 \in A^{e}$ is contained in the left-ideal generated by the set $\left\{1 \otimes b_{i}^{*}-b_{i} \otimes 1\right\}$.

1 An algebra $A$ over a ring $K$ will be called locally finite if every finitely generated subalgebra is contained in a finitely generated free $K$-submodule of $A$. 
Theorem 1 shows the existence of elements $y_{1}, \cdots, y_{k}$ such that $\left(1 \otimes b_{i}^{*}-b_{i} \otimes 1\right) \sum_{j} x_{j} \otimes y_{j}^{*}=O$ and $\sum_{j} x_{j} y_{j}=1$. Thus

$$
\left(1 \otimes z^{*}-z \otimes 1^{*}\right) \sum_{j} x_{j} \otimes y_{j}^{*}=0
$$

for every $z \in B$, that is,

$$
\sum z x_{j} \otimes y_{j}=\sum x_{j} \otimes y_{j} z
$$

If we write $z x_{j}$ as a linear combination of the $x_{i}$ 's, this formula shows that $y_{j} z$ is a linear combination of the $y$ 's, that is,

$$
y_{j} z=\sum_{i} k_{i j} y_{i}\left(k_{i j} \in K\right)
$$

hence, $z=\sum_{j} x_{j} y_{j} z=\sum_{i, j} k_{i j} x_{j} y_{i}$, and, then, $B$ is contained in the $K$ submodule generated by the set $\left\{x_{j} y_{i}\right\}$

Finally, if we write the elements $x_{j} y_{i}$ in terms of a basis, say $\left\{x_{k}\right\}$, since only a finite number of $x_{k}$ 's appear in each $x_{j} y_{i}, B$ is contained in a finitely generated $K$-free $K$-submodule of $A$.

4. Algebras over a field. In the case $K$ is a field, then, trivially, $A$ is a $K$-free $K$-module and, if w.dim $A=O$, the conditions of Theorems 1 and 2 must be satisfied.

The results of [3] (IX, 7.5 and 7.6), referred to weak dimension (i.e., starting from IX. 28 intead of IX. 2.8a) may be condensed, by using the equivalence between w.gl.dim $R=O$ and $R$ being a (von Neumann) regular ring [5], in the following proposition:

Proposition 1. If $A$ is a K-algebra over a regular ring $K$, then w.dim $A=O$ if and only if $A^{e}$ is a regular ring.

In the case of commutative algebras over a field a complete characterization of the case w.dim $A=O$ is obtained in the following result.

Theorem 3. Let $A$ be a commutative algebra over a field $K$. Then, then following conditions are equivalent:

(i) $A$ is locally separable ${ }^{2}$

(ii) w. $\operatorname{dim} A=O$

(iii) $A \otimes F$ is regular for every field $F$ containing $K$.

Proof. (i) $\Rightarrow$ (ii). Obviously, since $A$ is locally separable, it satisfies the conditions of Theorem 1.

(ii) $\Rightarrow$ (iii). This is a trivial consequence of the inequality

$$
\text { w.gl.dim } A \otimes F \leq \text { w.gl.dim } F+\text { w.dim } A
$$

obtained from the spectral sequences [3] (XVI, 5.5a p.347) and the equivalence between w.gl.dim $R=0$ and regularity obtained in [5].

${ }^{2}$ An algebra $A$ over a field $K$ is called locally separable if every finitely generated subalgebra is contained in a (finitely generated) separable subalgebra. 
(iii) $\Rightarrow$ (i). If $A$ is commutative and it is not locally finite over $K$, then there is at least one element $x$ which is transcendental over $K$, hence $A$ contains a subalgebra isomorphic with the polynomial ring $K[x]$.

For every polynomial $p(x)$, let $0_{p}$ be the set of elements $y \in A$ such that $y p(x)=0$. Let $I=\cup 0_{p}$, then, trivially, $I$ is an ideal in $A$, and no element of $K(x)$ is in $I$; otherwise, if $q(x) \in I$, there is a $p(x)$ such that $q(x) p(x)=O$, contradicting the transcendency of $x$.

If $A$ is regular, for every $p(x)$ there is an element $z$ such that $z(p(x))^{2}=p(x)$, hence $1-z p(x) \in I$ and, in $A / I$, the images of all $p(x)$ have inverses, so $A / I$ contains a subalgebra isomorphic with the field of rational functions $K(X)$.

Let us call $B=A / I$. If $A$ is regular, then $B$ is regular too, and, from the exactness of $A \rightarrow B \rightarrow 0$ we obtain $F \otimes A \rightarrow F \otimes B \rightarrow 0$ exact. Then, if $F \otimes A$ is regular, so is $F \otimes B$.

Since $B \supseteq K(X)$ and $K(X)$ is a field, $B$ is the direct sum of $K(X)$ modules isomorphic with $K(X)$, hence, from the fact that $\otimes$ distributes on direct sums, $F \otimes B$ is a direct sum of $F \otimes K(X)$-modules isomorphic with $F \otimes K(X)$. Applying now ([2], Prop. 2, p. 659), we obtain w.gl.dim $F \otimes$ $B \geq$ w.gl.dim $F \otimes K(X)$. Then, we must prove just that $F \otimes K(X)$ is not a regular ring. In fact, if $F$ is any field containing properly $K$, then $F \otimes K(X)$ contains a subring isomorphic with $F \otimes K[X] \approx F[X]$, which is an integral domain, and $F \otimes K(X)$ is the set of rational functions $q(x) / p(x)$ with $q(x) \in F[X]$ and $p(x) \in K[X]$, hence, it is an integral domain but not a field because it has no inverse for $q(x) \in F[X]$ if $q(x) \notin K[X]$, thus $F \otimes K(X)$ is not a regular ring.

Thus, condition (iii) implies $A$ is locally finite.

Since $A \otimes F$ is regular and commutative, $B \otimes F$ has to be semisimple for every finitely generated subalgebra $B$ and every field $F$ containing $K$, hence $B$ is separable and so $A$ is locally separable.

The result of Corollary 2 can be extended, in the case of algebras over a field, by using the following result of Kaplansky ([7], Lemma 1).

LEMma 3. If I is a countably generated left-ideal in a regular ring $R$, then $\operatorname{dim}_{k} I=O$.

A direct implication of this lemma is obtained in

Theorem 4. Let $A$ be an algebra over a field $K$. If $[A: K]=\aleph_{0}$ and w.dim $A=O$, then $\operatorname{dim} . A=1$.

Proof. Since w.dim $A=O$ implies $A \otimes A^{*}$ regular, and $\operatorname{Ker}\left(A^{e} \rightarrow A\right)$ is generated by the set $\left\{x_{i} \otimes 1-1 \otimes x_{i}^{*}\right\}$ (where the $x_{i}{ }^{\prime}$ s are generators of $A$ ) and this set is countable, then $\operatorname{Ker}\left(A^{e} \rightarrow A\right)$ is projective. Thus, $\operatorname{dim} A \leq 1$. Since $\operatorname{dim} A=O$ implies $[A ; K]$ finite, then $[A: K]=\aleph_{0}$ implies $\operatorname{dim} A=1$. 
We shall say that an algebra $A$ is locally one dimensional if every finite set of elements is contained in a subalgebra $B$ such that $\operatorname{dim} B \leq 1$. The following theorem approximates the result obtained in Theorem 3 for commutative algebras.

TheOREM 5. Let $A$ be an algebra over a field $K$. If $\mathrm{w} \cdot \operatorname{dim} A=O$, then $A$ is locally one dimensional.

Proof. Let $\left\{a_{1}, \cdots, a_{n}\right\}$ be a finite set of elements in $A$. Let $B_{0}$ be the subalgebra generated by this set. Since $A^{e}$ is regular, there is an idempotent $e_{1}$ such that $\left(1 \otimes a_{i}^{*}-a_{i} \otimes 1\right) e_{1}=O$ and $e_{1}$ is mapped onto 1 by the natural map $\sigma: A^{e} \rightarrow A$ (In fact, the left ideal generated by the finite set $\left\{1 \otimes a_{i}^{*}-a_{i} \otimes 1\right\}$ is generated by an idempotent $1-e_{1}$ which is mapped onto 0 ).

If $x \in B$, then $1 \otimes x^{*}-x \otimes 1^{*}=\sum y_{i}\left(1 \otimes a_{i}^{*}-a_{i} \otimes 1\right)\left(y_{i} \in A^{e}\right)$, thus $\left(1 \otimes x^{*}-x \otimes 1^{*}\right) e_{1}=O$ for every $x \in B_{0}$.

Let $\left\{b_{1}, \cdots, b_{m}\right\}$ be the set of elements of $A$ appearing in $e_{1}$, and $B_{1}$ the subalgebra generated by $\left\{a_{1}, \cdots, a_{n}, b_{1}, \cdots, b_{m}\right\}$. Then, by the same arguments, there is an idempotent $e_{2} \in A^{e}$ such that $(x \otimes 1-1 \otimes$ $\left.x^{*}\right) e_{2}=O$ for every $x \in B_{1}$, and $\sigma\left(e_{2}\right)=1$.

By repeating the process we obtain a chain of subalgebras $B_{0} \subseteq$ $B_{1} \subseteq B_{2} \subseteq \cdots$ If we call $B=\cup B_{i}(i=1,2, \cdots)$ then Theorem 1 implies $\mathrm{w} \cdot \operatorname{dim} B=0$. In fact, for every finite subset $\left\{x_{1}, \cdots, x_{h}\right\}$ in $B$ there is a finitely generated subalgebra $B_{k}$ with $x_{i} \in B_{k}(1 \leq i \leq h)$, then $e_{k+1}$ satisfies $\left(x_{i} \otimes 1^{*}-1 \otimes x_{i}^{*}\right) e_{k+1}=0, \sigma\left(e_{k+1}\right)=1$, and $e_{k+1} \in B^{e}$.

Since $B$ is, at most, countably generated, then Corollary 2 and Theorem 4 imply $\operatorname{dim} B \leq 1$.

Remark 1. According to Proposition 1, if $A$ is an algebra over a regular $\operatorname{ring} K, \mathrm{w} \cdot \operatorname{dim} A=0$ implies $A^{e}$ is regular. Then, in this case, Theorem 4 may be expressed in the following way:

Theorem 4'. Let $A$ be an algebra over a regular ring $K$. If $A$ is denumerably generated and w.dim $A=0$, then $\operatorname{dim} A \leq 1$.

Thus, Theorem 5 is valid for algebras over a regular ring $K$.

5. Algebras with descending chain condition. Theorem 3 shows that, for a commutative algebra over a field, w.dim $A=0$ if and only if $A$ is locally separable. We do not know whether this statement is true in the non commutative case.

In the case of algebras satisfying the descending chain condition for left-ideals, the following result, suggested to the author by Professor Rosenberg, characterizes completely the $0-\mathrm{w}$. dimensional case. 
Theorem 6. Let $A$ be an algebra over a field $K$ satisfying the descending chain condition for left ideals. Then, w.dim $A=0$ if and only if :

(a) $A$ is semisimgle

(b) $A$ is locally finite over $K$

(c) The center $C$ of $A$ is locally separable.

Proof. If $w \cdot \operatorname{dim} A=0$, condition (a) follows from the regularity of $A$, (b) from Theorem 1 and (c) from Theorem 3.

Suppose, now $A$ satisfies (a), (b) and (c). Since $A$ is semisimple, it is a direct sum of (a finite number of) simple algebras $S_{i}$ satisfying conditions (b) and (c), and, because of the direct sum decomposition, w.dim $A=\max \left(\mathrm{w} \cdot \operatorname{dim} S_{i}\right)$. Since each $S_{i}$ is a matrix ring over a division algebra $D_{i}$ satisfying (b) and (c) and w.dim $S_{i}=\mathrm{w} \cdot \operatorname{dim} D_{i}$, it will be enough to prove the sufficiency of these conditions for division algebras.

Let $A$ be a division algebra. Condition (c) implies w.dim $C=O$. According to the sub-additivity of the dimension ([9], Th. 5, p.93) we have w.dim $A \leq \mathrm{w} \cdot \operatorname{dim} . C+C$-w.dim $A$, then, it is sufficient to prove that $A \otimes_{0} A^{*}$ is regular. This is so if $A \otimes_{C} S^{*}$ is regular for every finitely generated subalgebra $S$ of $A$.

Since $A^{*}$ is locally finite and $S^{*}$ finitely generated, then $\left[S^{*}: C\right]<\infty$ and $S^{*}$ is a division ring. Thus $A \otimes_{z} S^{*}$ satisfies the descending chain condition. Since $A$ is central simple and $S^{*}$ simple (because now we are considering $A$ and $S$ as algebras over $C$ ), then $A \otimes_{0} S^{*}$ is simple, hence regular, and the theorem is proved.

6. Group algebras. In [2], Auslander studies necessary and sufficient conditions for a group $G$ and a ring $K$ to obtain (von Neumann) regular group algebras $K(G)$. He proved the necessity of $G$ being a torsion group and the sufficiency of $G$ being locally finite, besides the conditions on $K$.

In Theorem 8 we prove the necessity of the local finiteness, and then regular group algebras are completely characterized.

A similar difference existed between Theorem 3 and 4 in [8], but by direct aplication of Theorem 2 we fill the gap obtaining the following result.

Theorem 7. Let $G$ be a group, $S$ a subgroup contained in the center of $G$ and $K$ any commutative ring, then $K(S)$-w.dim $K(G)=O$ if and only if $G / S$ is locally finite and $K$ is uniquely divisible by the order of each element in $G / S$.

In fact, the local finiteness of $K(G)$ as a $K(S)$-algebra implies the local finiteness of $G / S$. 
THeorem 8. Let $G$ be a group and $K$ any commutative ring. Then $K(G)$ is regular if and only if $G$ is locally finite and $K$ is a regular ring uniquely divisible by the order of each element in $G$.

Proof. A trivial modification in the proof of ([3] X. 6.1) may be used to prove

$$
\mathrm{w} \cdot \operatorname{dim} K(G)=\mathrm{r} \cdot \mathrm{w} \cdot \operatorname{dim}_{K(G)} K .
$$

Thus, $K(G)$ regular implies w.dim $K(G)=O$ and Theorem 2 implies $G$ is locally finite.

The remaining part of the proof follows from Auslander's result. It also may be seen as a special case of Theorem 7 .

REMARK 2. The proof of the necessity of the local finiteness of $G$ for a group algebra $K(G)$ to be regular does not need all the homological machinery. In fact, it follows immediately from the following lemma:

Lemma 4. Let $K(G)$ be the group algebra generated by a group $G$ over any commutative ring $K$ and $g_{1}, \cdots, g_{n}$ be elements of $G$. Then the subgroup $S$ generated by $\left\{g_{1}, \cdots, g_{n}\right\}$ is finite if and only if there is an element $x \in K(G)$ such that $\left(1-g_{i}\right) x=0(1 \leq i \leq n)$. If this is the case, $x=s y$, where $s$ is the sum of all elements in $S$.

Proof. If $S$ is finite, the sum $s$ of all elements in $S$ satisfies the equations $\left(1-g_{i}\right) x=0$, and so every product $s y$.

Conversely, suppose $\left(1-g_{i}\right) x=0(1 \leq i \leq n)$. Thus, $x=g_{1} x=\cdots=$ $g_{n} x$. Since every $f \in S$ is a product of powers of the $g_{i}$ 's, then $f x=x$. Let $x=\sum_{1}^{m} k_{j} h_{j}\left(h_{j} \in G\right)$. For every $f \in S, f x=x$ implies $x$ has a term $k_{1} f h_{1}$ and so all elements of $S$ appear multiplied by $k_{1} h_{1}$, hence $S$ is finite (because $x$ is a finite sum). Furthermore, we obtained $x=k_{1} s h_{1}+x^{\prime}$, with $\left(1-g_{i}\right) x^{\prime}=0$. By induction on the number of terms in $x$ we obtain the last result.

A complete proof of Theorem 8 may be obtained as follows: Suppose $K(G)$ is regular. Then the ring homomorphism $\sigma: K(G) \rightarrow K$ defined by the group homomorphism $G \rightarrow\{1\}$ implies $K$ is regular.

If $K(G)$ is regular, every finitely generated proper left ideal is a direct summand, hence it is annihilated, on the right, by a non-zero element $x \in K(G)$. Since all $1-g$ are in $\operatorname{Ker} \sigma$, every finite set generates a proper left ideal, and so the previous lemma implies $G$ is locally finite.

Suppose $g \in G$ has order $n$. By Lemma 1 there is an element $x$ with $\sigma(x)=1$ and $(1-g) x=O$, hence Lemma 4 implies $x=s y(s=$ $\sum g^{i}$ ) and so $\sigma(x)=\sigma(s) \sigma(y)=n \sigma(y)=1$, hence $n$ has an inverse in $K$ 
and the necessity of the conditions is proved.

Suppose, now, $K$ and $G$ satisfy the conditions of the theorem. Let $x=\sum k_{i} g_{i} \in \operatorname{Ker} \sigma$, so, $x=\sum k_{i}\left(g_{i}-1\right)$. Let $S$ be the subgroup generated by $g_{1}, \cdots, g_{n}, m$ its order and $s$ the sum of all elements in $S$. Since $m$ has an inverse $m^{-1}$ in $K$, then $y=m^{-1} s$ satisfies $x y=O, \sigma(y)=$ 1 , and Lemma 1 implies $K$ is $K(G)$-flat. So w.dim $K(G)=\mathrm{w} \cdot \operatorname{dim}_{K(G)} K=$ $O([3] \mathrm{X}, 6.2)$ and $K(G)$ is regular.

7. Weak dimension and Jacobson semisimplicity. A ring will be called $J$-semisimple if its Jacobson radical is $(O)$.

If $T$ is a ring and $M$ a left- $T$-module, then the $\operatorname{ring} \operatorname{Hom}_{T}(M, M)$, with the operations defined in the classical way is a topological ring by defining the finite topology induced by $M$. ([6], Ch. IV).

If we are in the situation $S \subseteq R \subseteq \operatorname{Hom}_{T}(M, M)$, where $S$ and $R$ are rings, we shall say $S$ is dense in $R$ if it is so in the finite topology induced by $\operatorname{Hom}_{T}(M, M)$.

In this section we shall prove the following theorem:

Theorem 9. Let $A$ be a K-projective $K$-algebra. If $B$ is $J$-semisimple K-algebra and w.dim $A=0$, then $A \otimes B$ is J-semisimple.

Before proving the Theorem we shall state the following lemmas:

Lemma 5. Let $T$ be any ring and $M$ a left-T-module. If $S, R$ are rings such that $S \subseteq R \subseteq \operatorname{Hom}_{T}(M, M), R$ is regular and $S$ is dense in $R$, then $S$ is J-semisimple. ${ }^{3}$

Proof. Let $x \in S$. Since $R$ is regular, there are elements $y, z \in R$, $z \neq O$, such that $z(1-x y)=O$. Since $R \subseteq \operatorname{Hom}_{T}(M, M)$, there is at least one $m \in M$ such that $m^{z} \neq O$ and $m^{z(1-x y)}=O$, that is, there exists an $n \in M\left(n=m^{z}\right)$ such that $n \neq O$ and $n=n^{2 y}$. Now, we have $x \in S$, $y \in R,\left(n^{x}\right)^{y}=n$, and $S$ is dense in $R$, then there is an $u \in S$ such that $\left(n^{x}\right)^{u}=n$, that is, $n^{1-x u}=O$, and $1-x u$ can not have an inverse in $S$, so $x u$ is not quasi-regular, and $S$ is $J$-semisimple.

Lemma 6. If $A$ is a $K$-projective K-algebra and $B$ is a K-algebra which is a subdirect sum of $K$-algebras $P_{i}$, then $A \otimes B$ is a subdirect sum of $A \otimes P_{i}$.

Proof. $B$ is a subdirect sum of $P_{i}^{\prime}$ 's if and only if the sequences $B \rightarrow P_{i} \rightarrow 0$ and $0 \rightarrow B \rightarrow \Pi P_{i}$ are exact.

3 The conditions of the lemma are, evidently, stronger that those which are really needed in the proof. In fact, we only need $S$ to be 1 -fold transitive in $R$ and $R J$-semisimple in which, for every element $x$ there is an $y$ such that 1-xy has a left annihilator.

It may be secn that, if $S$ is commutative, the conditions of Lemma 5 are necessary. 
Now from the exact sequence $B \rightarrow P_{i} \rightarrow 0$ we obtain $A \otimes B \rightarrow$ $A \otimes P_{i} \rightarrow 0$ exact. We need only to prove the exactness of $0 \rightarrow A \otimes B \rightarrow$ $\Pi\left(A \otimes P_{i}\right)$.

Since $A$ is $K$-projective, we have, from $0 \rightarrow B \rightarrow \Pi P_{i}$, the exact sequence $0 \rightarrow A \otimes B \rightarrow A \otimes \Pi P_{i}$. We have ([3], Ex. II. 2, 31) a natural homomorphism

$$
A \otimes \Pi P_{i} \rightarrow \Pi\left(A \otimes P_{i}\right)
$$

which is, trivially, a monomorphism if $A=K$. Since $\otimes$ commutes with direct sums, it is a monomorphism if $A$ is $K$-free and, a posteriori, if $A$ is $K$-projective. Then the composite map gives the exact sequence

$$
0 \rightarrow A \otimes B \rightarrow \Pi\left(A \otimes P_{i}\right)
$$

and the lemma is proved.

Proof of the theorem. Since $B$, being semisimple, is a subdirect sum of primitive rings $P_{i}$, then the previous lemma implies that $A \otimes B$ is a subdirect sum of rings $A \otimes P_{i}$; then, to prove the theorem it is sufficient to show that the rings $A \otimes P_{i}$ are $J$-semisimple. Now, since $P_{i}$ is primitive, it is dense in a ring of linear transformations, that is, $P_{i} \subseteq R_{i}=\operatorname{Hom}_{F_{i}}\left(M_{i}, M_{i}\right)$ where the rings $R_{i}$ are regular and the $P_{i}$ 's are dense in the $R_{i}$ 's. Since $A$ is $K$-projective, we may apply the spectral sequences [3] (XVI, 5a, p. 347) and then, $R_{i}$ regular and w. $\operatorname{dim} A=O$ give $A \otimes R_{i}$ regular.

If we show the inclusion $A \otimes P_{i} \subseteq A \otimes R_{i} \subseteq \operatorname{Hom}_{A \otimes F_{i}}\left(A \otimes M_{i}, A \otimes M_{i}\right)$ and the density of $A \otimes P_{i}$ into $A \otimes R_{i}$, Lemma 5 completes the proof of the theorem.

Since $A$ is $K$-projective, we have the exact sequence $0 \rightarrow A \otimes S_{i} \rightarrow$ $A \otimes R_{i}=A \otimes \mathrm{Ho}_{F} \mathrm{~m}\left(M_{i}, M_{i}\right)$.

If $A$ is $K$-free, the natural mapping $A \otimes \operatorname{Hom}_{F}\left(M_{i}, M_{i}\right) \rightarrow \operatorname{Hom}_{F^{F}}(M$, $\left.A \otimes M_{i}\right)$ is the natural mapping of a direct sum into a direct product, which is a monomorphism. Since $A$, being projective, is a direct summand of a free module, and since both $\otimes$ and Hom commute with finite direct sums, then the given mapping is also a monomorphism.

From the natural isomorphism $\operatorname{Hom}_{F}\left(M_{i}, A \otimes M_{i}\right) \approx \operatorname{Hom}_{A \otimes r}\left(A \otimes M_{i}\right.$, $A \otimes M_{i}$ ) we obtain the inclusions.

$$
A \otimes S_{i} \subseteq A \otimes R_{i} \subseteq \operatorname{Hom}_{A \otimes F_{i}}\left(A \otimes M_{i}, A \otimes M_{i}\right)
$$

Let $x \in A \otimes R_{i}$, then $x=\sum a_{j} \otimes r_{j}$, and $v_{k}=\sum_{l} b_{k l} \otimes m_{k l}\left(b_{k l} \in A\right.$, $\left.m_{k l} \in M_{i}\right)$ be a finite set of elements in $A \otimes M_{i}$. Then $x\left(v_{i}\right)=\sum_{l, j} a_{j} b_{k l} \otimes$ $r_{j}\left(m_{k l}\right)$. Since the set $\left\{m_{k l}\right\}$ is finite and $B_{i}$ is dense in $R_{i}$, for each $r_{j}$ there is an $s_{j} \in B_{i}$ such that $r_{j}\left(m_{k l}\right)=s_{j}\left(m_{k l}\right)$, then $y=\sum a_{j} \otimes s_{j} \in A \otimes P_{i}$ and $y\left(v_{k}\right)=x\left(v_{k}\right)$, so $A \otimes P_{i}$ is dense in $A \otimes R_{i}$. 
As a consequence of this theorem we can state the following corollary :

Corollary 3. If $A, B$ are algebras over a field $K$ and $w \cdot \operatorname{dim} B=O$, Then $J(A \otimes B)=J(A) \otimes B$. (We call $J(R)$ the Jacobson radical of a ring $R)$.

In fact, since $A / J(A)$ is semisimple, from the exact sequence

$$
0 \rightarrow J(A) \otimes B \rightarrow A \otimes B \rightarrow(A / J(A)) \otimes B \rightarrow 0
$$

we obtain $J(A) \otimes B \supseteq J(A \otimes B)$.

From Theorem 2 and ([6], V. 14, Th. 1, p 123) it follows that every element in $J(A) \otimes B$ is quasi-regular, so it is a radical ideal in $A \otimes B$. Thus $J(A) \otimes B \subseteq J(A \otimes B)$ and the corollary is proved.

This result generalizes ([1], Th. 1).

\section{REFERENCES}

1. S. A. Amitsur, The radical of field extensions, Bull. of the Research Council of Israel, 7F (1957), 1-10.

2. M. Auslander, On regular group rings, Proc. Amer. Math. Soc., 8 (1957), 658-664.

3. H. Cartan and S. Eilenberg, Homological Algebra, Princeton University Press, 1956.

4. M. Harada, The weak dimension of algebras and its applications, J. Inst. Polytechnics, Osaka City University 9 (1958), 47-58.

5. - Note on the dimension of modules and algebras, J. Inst. Polytechnics, Osaka City University 7 (1956), (17-27).

6. N. Jacobson, Structure of rings, Amer. Math. Soc., Coll. Pub., XXXVII, 1956.

7. I. Kaplansky, On the dimension of modules and algebras, Nagoya Math. Journal, 13 (1938), 85-89.

8. J. E. McLaughlin, A note on regular group rings, Mich. Math. Jour., 5 (1958), 127-128.

9. A. Rosenberg and D. Zelinsky, Cohomology of infinite algebras, Trans. Amer. Math. Soc., 82 (1956), 85-98.

10. O. E. Villamayor, On the semisimplicity of group algebras, Proc. Amer. Math. Soc., 9 (1958), 621-627.

Universidad Nacional de la Plata

The Institute For Advanced Study

NORTHWESTERN UNIVERSITY 



\section{PACIFIC JOURNAL OF MATHEMATICS}

\section{EDITORS}

\section{David Gilbarg}

Stanford University Stanford, California

\section{R. A. Beaumont}

University of Washington

Seattle 5, Washington

\section{A. L. Whiteman}

University of Southern California Los Angeles 7, California

L. J. Paige

University of California

Los Angeles 24, California

\section{ASSOCIATE EDITORS}

E. F. BECKENBACH
C. E. BURGESS
E. HEWITT
A. HORN

A. HORN

\author{
V. GANAPATHY IYER \\ R. D. JAMES \\ M. S. KNEBELMAN \\ L. NACHBIN
}

I. NIVEN

T. G. OSTROM

H. L. ROYDEN

M. M. SCHIFFER
E. G. STRAUS

G. SZEKERES

F. WOLF

K. YOSIDA

\section{SUPPORTING INSTITUTIONS}

\author{
UNIVERSITY OF BRITISH COLUMBIA \\ CALIFORNIA INSTITUTE OF TECHNOLOGY \\ UNIVERSITY OF CALIFORNIA \\ MONTANA STATE UNIVERSITY \\ UNIVERSITY OF NEVADA \\ OREGON STATE COLLEGE \\ UNIVERSITY OF OREGON \\ OSAKA UNIVERSITY \\ UNIVERSITY OF SOUTHERN CALIFORNIA
}

\author{
STANFORD UNIVERSITY \\ UNIVERSITY OF TOKYO \\ UNIVERSITY OF UTAH \\ WASHINGTON STATE COLLEGE \\ UNIVERSITY OF WASHINGTON \\ * * * * \\ AMERICAN MATHEMATICAL SOCIETY \\ CALIFORNIA RESEARCH CORPORATION \\ HUGHES AIRCRAFT COMPANY \\ SPACE TECHNOLOGY LABORATORIES
}

Mathematical papers intended for publication in the Pacific Journal of Mathematics should be typewritten (double spaced), and the author should keep a complete copy. Manuscripts may be sent to any one of the four editors. All other communications to the editors should be addressed to the managing editor, L. J. Paige at the University of California, Los Angeles 24, California.

50 reprints per author of each article are furnished free of charge; additional copies may be obtained at cost in multiples of 50 .

The Pacific Journal of Mathematics is published quarterly, in March, June, September, and December. The price per volume (4 numbers) is $\$ 12.00$; single issues, $\$ 3.50$. Back numbers are available. Special price to individual faculty members of supporting institutions and to individual members of the American Mathematical Society: $\$ 4.00$ per volume; single issues, $\$ 1.25$.

Subscriptions, orders for back numbers, and changes of address should be sent to Pacific Journal of Mathematics, 2120 Oxford Street, Berkeley 4, California.

Printed at Kokusai Bunken Insatsusha (International Academic Printing Co., Ltd.), No. 6, 2-chome, Fujimi-cho, Chiyoda-ku, Tokyo, Japan.

PUBLISHED BY PACIFIC IOURNAL OF MATHEMATICS, A NON-PROFIT CORPORATION

The Supporting Institutions listed above contribute to the cost of publication of this Journal, but they are not owners or publishers and have no responsibility for its content or policies. 


\section{Pacific Journal of Mathematics}

\section{Vol. 9, No. $3 \quad$ July, 1959}

Errett Albert Bishop, A minimal boundary for function algebras . . . . . . . . . . . . 629

John W. Brace, The topology of almost uniform convergence . . . . . . . . . . . . 643

Cecil Edmund Burgess, Chainable continua and indecomposability .......... 653

L. Carlitz, Multiplication formulas for products of Bernoulli and Euler

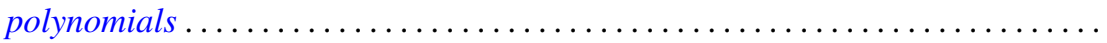

Eckford Cohen, A class of residue systems (mod $r$ ) and related arithmetical

functions. II. Higher dimensional analogues ....................

Shaul Foguel, Boolean algebras of projections of finite multiplicity . . . . . . . . . .

Richard Robinson Goldberg, Averages of Fourier coefficients .................

Seymour Goldberg, Ranges and inverses of perturbed linear operators .

Philip Hartman, On functions representable as a difference of convex functions ....

Milton Vernon Johns, Jr. and Ronald Pyke, On conditional expectation and

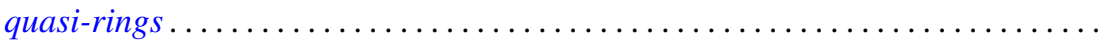

Robert Jacob Koch, Arcs in partially ordered spaces ....................

Gregers Louis Krabbe, A space of multipliers of type $L^{p}(-\infty, \infty) \ldots \ldots \ldots \ldots$

John W. Lamperti and Patrick Colonel Suppes, Chains of infinite order and their

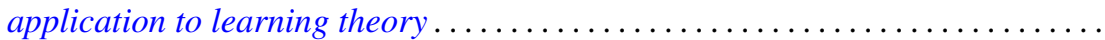

Edith Hirsch Luchins, On radicals and continuity of homomorphisms into Banach

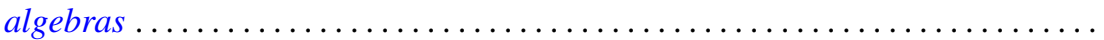

T. M. MacRobert, Multiplication formulae for the E-functions functions of their parameters.

Michael Bahir Maschler, Classes of minimal and representative domains and their kernel functions.

William Schumacher Massey, On the imbeddability of the real projective spaces in Euclidean space.

Thomas Wilson Mullikin, Semi-groups of class $\left(C_{0}\right)$ in $L_{p}$ determined by parabolic differential equations

Steven Orey, Recurrent Markov chains

Ernest Tilden Parker, On quadruply transitive groups ........ . .

Calvin R. Putnam, On Toeplitz matrices, absolute continuity, and unitary

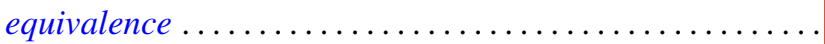

Helmut Heinrich Schaefer, On nonlinear positive operators.

Robert Seall and Marion Wetzel, Some connections between continued fractions and convex sets

Robert Steinberg, Variations on a theme of Chevalley

Olga Taussky and Hans Zassenhaus, On the similarity transformation between a

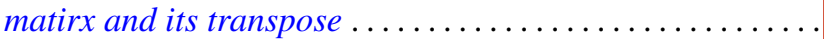

Emery Thomas, The suspension of the generalized Pontrjagin cohomology

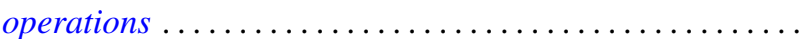

Joseph L. Ullman, On Tchebycheff polynomials ..................... 913

Richard Steven Varga, Orderings of the successive overrelaxation scheme ........ 925

Orlando Eugenio Villamayor, Sr., On weak dimension of algebras . 